Supporting Information for:

Size-Dependent Electroporation of Dye-Loaded Polymer Nanoparticles for Efficient and Safe Intracellular Delivery

Sylvie Egloff, Anne Runser, Andrey Klymchenko, ${ }^{*}$ Andreas Reisch*

Université de Strasbourg, CNRS, Laboratoire de Bioimagerie et Pathologies UMR 7021, F-67000 Strasbourg, France

*Corresponding authors : andrey.klymchenko@unistra.fr and reisch@unistra.fr 


\section{Optimization of Electroporation Conditions}

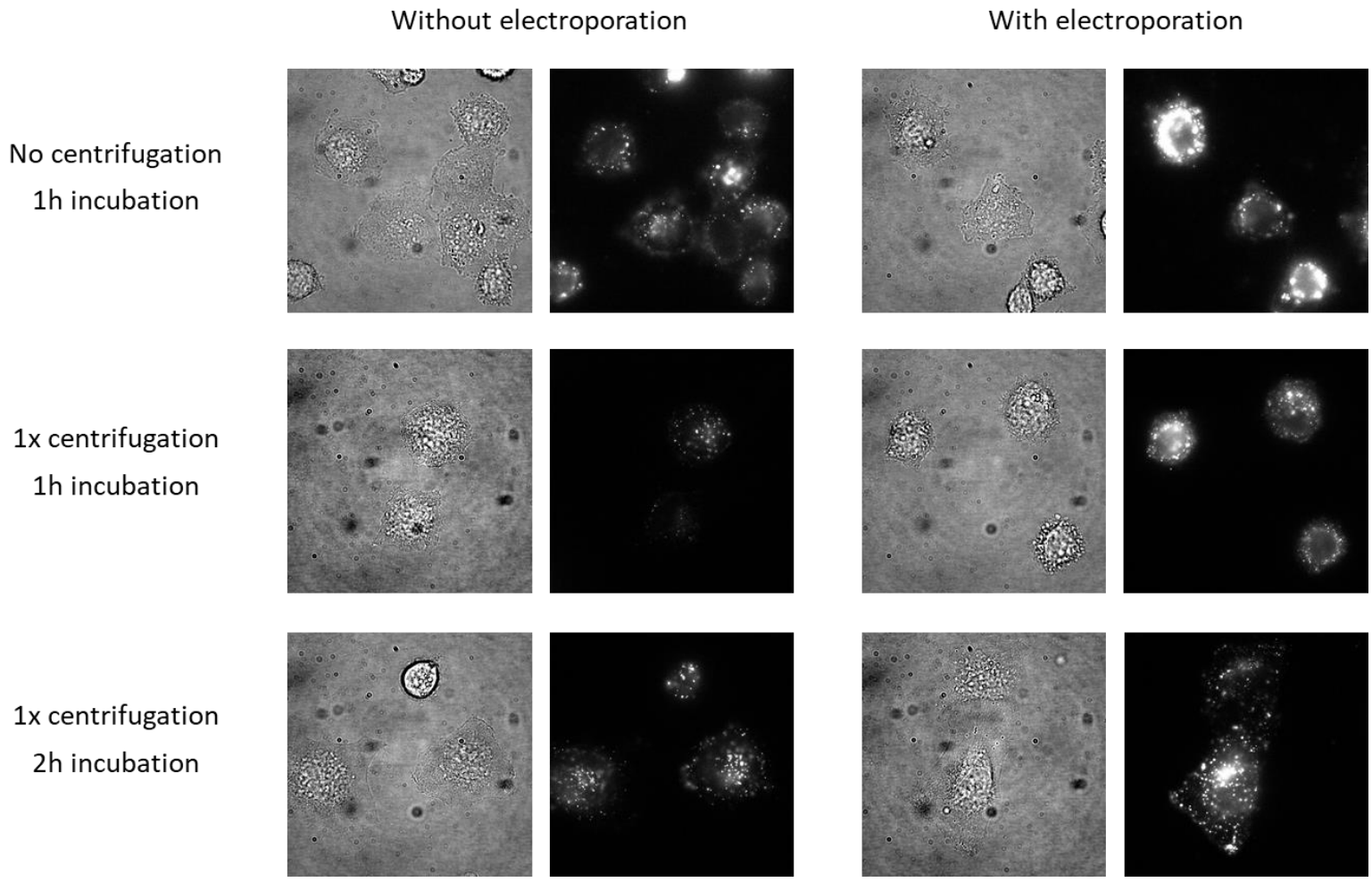

Figure S1. Influence of centrifugation and incubation time. DIC (left) and fluorescence (right) micrographs of cells treated with $13 \mathrm{~nm}$ NPs. The left and right columns correspond to conditions without and with electroporation, respectively. The effect of a centrifugation step directly after electroporation or bringing the cells in contact with the NP solution and of the incubation time following this treatment is evaluated. $\lambda_{\mathrm{ex}}=550 \mathrm{~nm}, \lambda_{\mathrm{em}}=555-635 \mathrm{~nm}$. Scale bars: $20 \mu \mathrm{m}$.

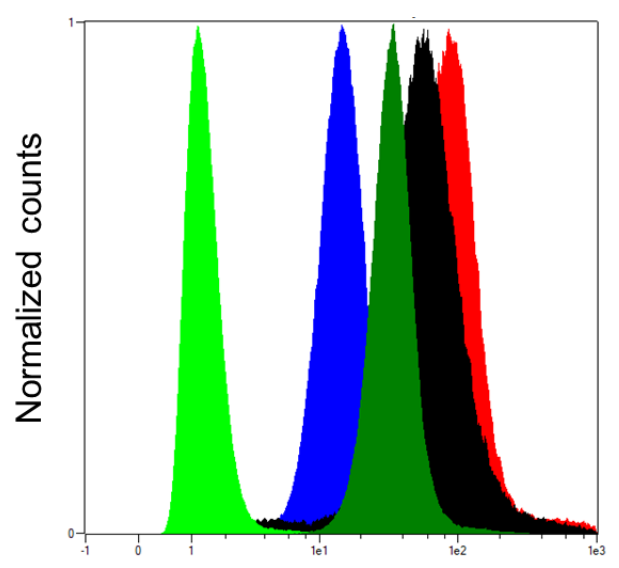

Fluorescence intensity (au)
Cells without NPs

Buffer R, 20 mg/L T80, no-EP, 1x centrifugation

Buffer R, $20 \mathrm{mg} / \mathrm{L}$ T80, EP, 1x centrifugation

Buffer R, 20 mg/L T80, no-EP, 2x centrifugation

Buffer R, 20 mg/L T80, EP, 2x centrifugation

Figure S2. Influence of a second centrifugation step as determined using flow cytometry. Cells were treated with $13 \mathrm{~nm}$ NPs with and without electroporation, followed directly by 1 or 2 centrifugation steps. The cells were then immediately analyzed using flow cytometry with a $561 \mathrm{~nm}$ for excitation and a $651 / 671 \mathrm{~nm}$ band pass filter for the emission. 
Without electroporation

DMEM with FBS

$1 x$ centrifugation

$2 \mathrm{~h} 30$ incubation

OptiMEM

$1 \mathrm{x}$ centrifugation

$2 \mathrm{~h} 30$ incubation
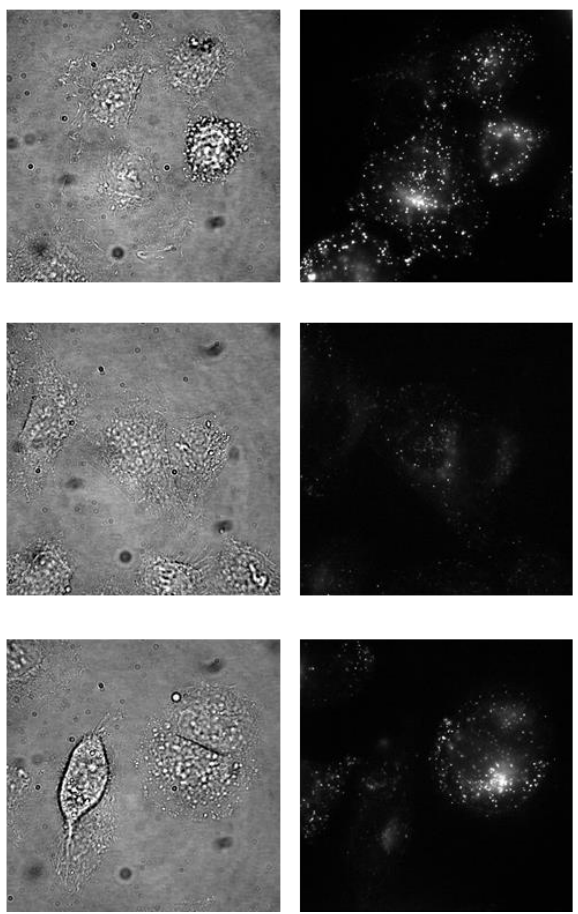

With electroporation
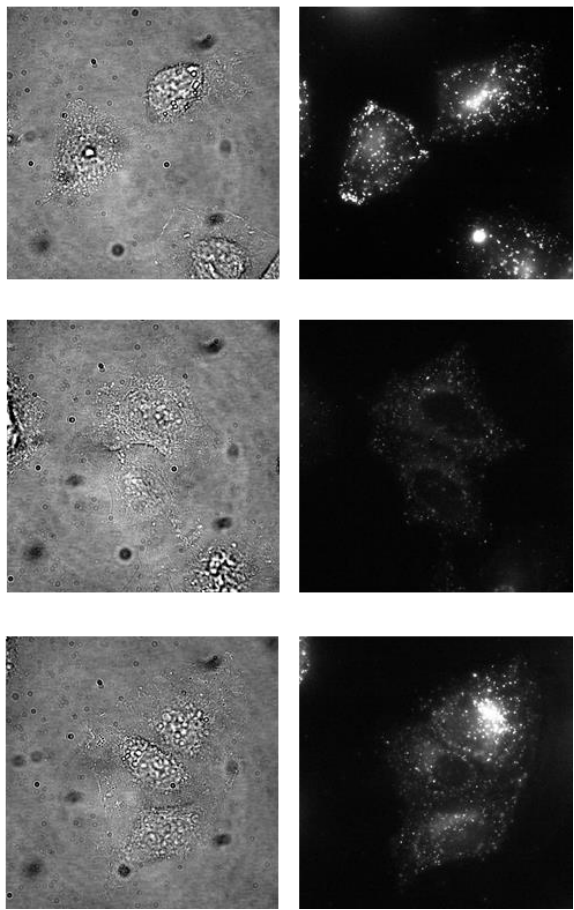

Figure S3. Effect of the electroporation medium. DIC (left) and fluorescence (right) micrographs of cells treated with $13 \mathrm{~nm}$ NPs. The left and right columns correspond to conditions without and with electroporation, respectively. The effect of different media was evaluated, in which the cells were brought in contact with the NP solution, and, if applicable, electroporated. DMEM with FBS gave lower signals for both, electroporated and non-electroporated cells. OptiMEM gave larger cell to cell variations. $\lambda_{\mathrm{ex}}=550 \mathrm{~nm}, \lambda_{\mathrm{em}}=555-635 \mathrm{~nm}$. Scale bars: $20 \mu \mathrm{m}$. 


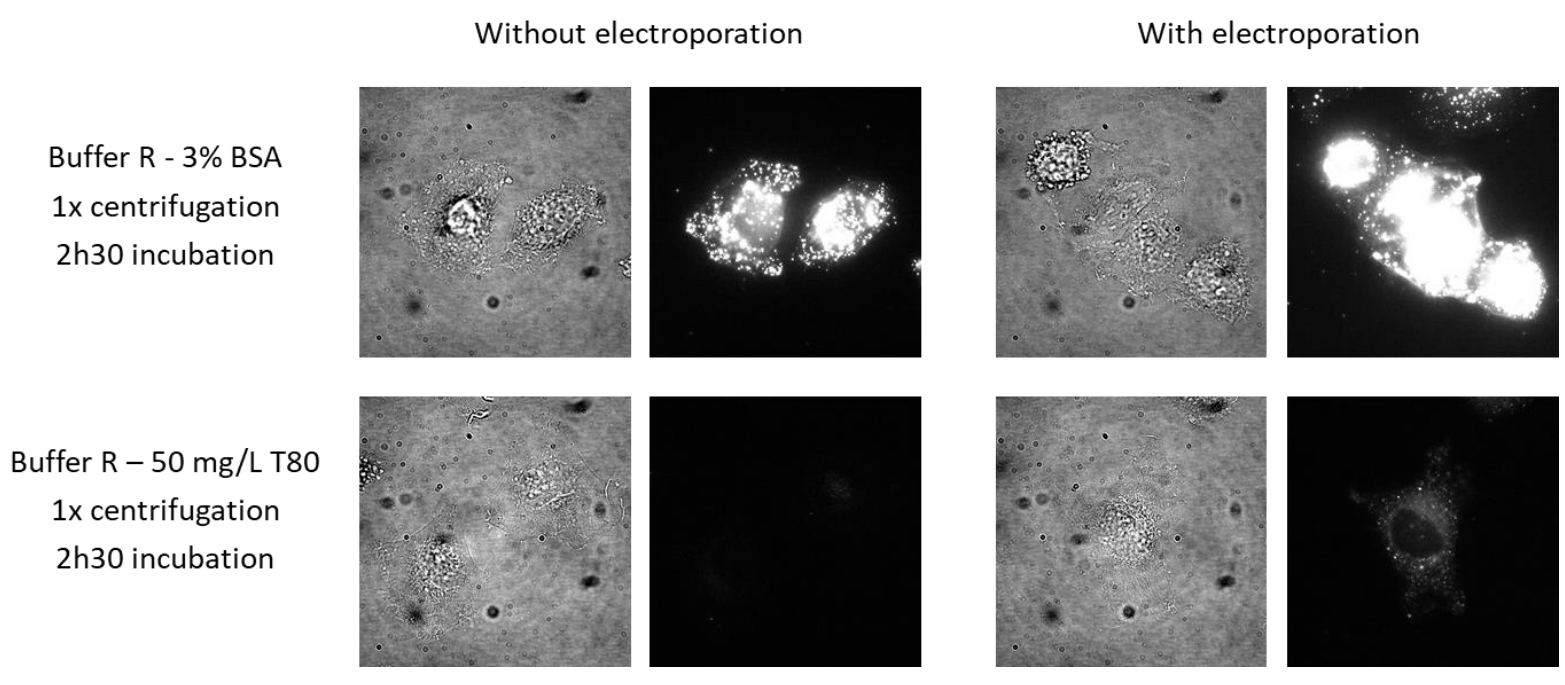

Figure S4. Control of interactions. DIC (left) and fluorescence (right) micrographs of cells treated with $13 \mathrm{~nm}$ NPs. The left and right columns correspond to conditions without and with electroporation, respectively. The effect of two blocking agents, bovine serum albumin (BSA) and Tween 80 (T80), added to the medium in which the cells were brought in contact with the NP solution, and, if applicable, electroporated, was evaluated. Tween 80 led to a strong reduction of NP endocytosis in the absence of electroporation. $\lambda_{\text {ex }}=550 \mathrm{~nm}, \lambda_{\mathrm{em}}=555-635 \mathrm{~nm}$. Scale bars: $20 \mu \mathrm{m}$. 


\section{Single Particle Tracking}
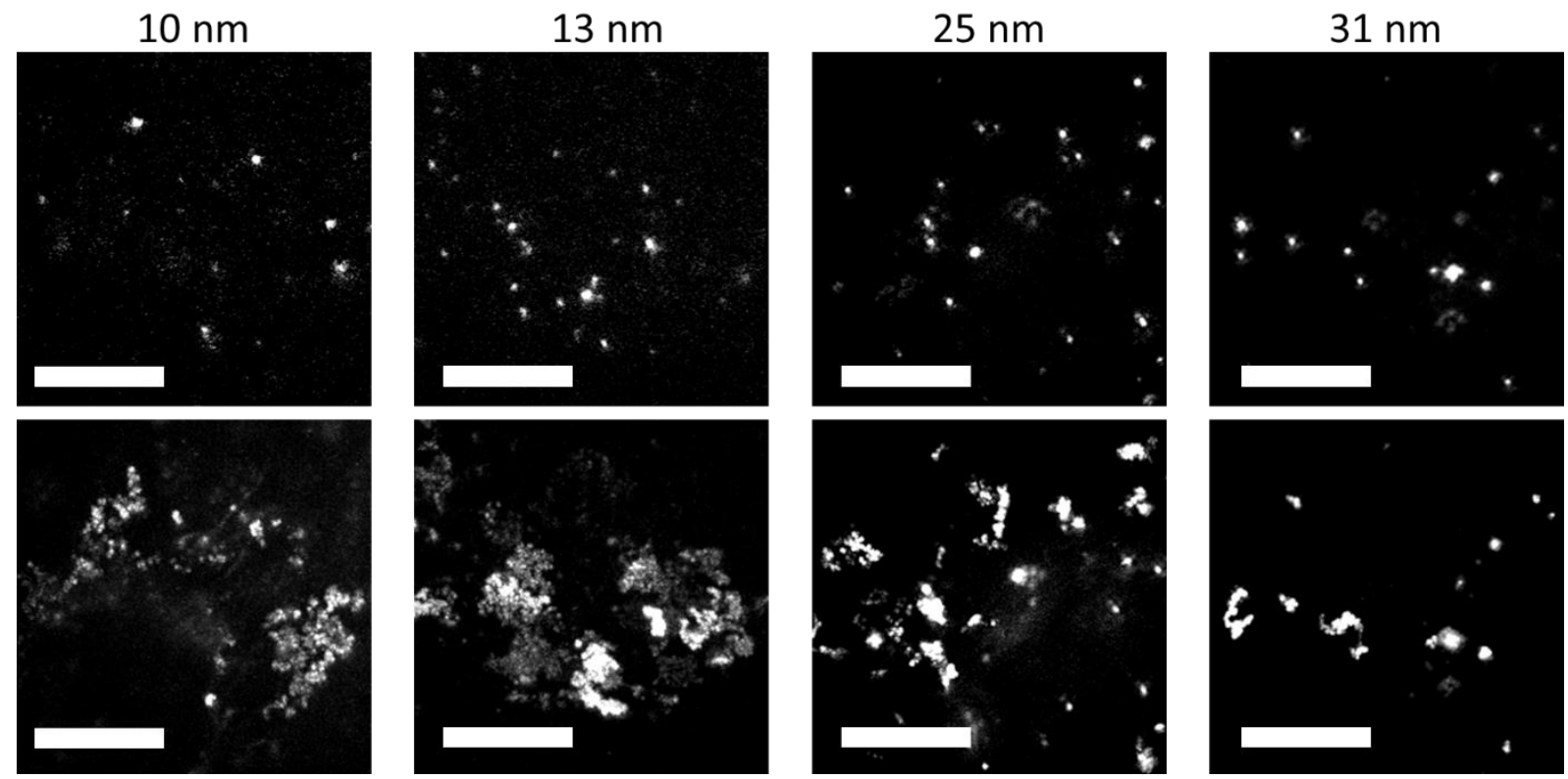

Figure S5. Examples of fluorescence micrographs of cells electroporated with NPs of different sizes used for single-particle tracking. Top: Instantaneous fluorescence. Bottom: Maximum projections over $30 \mathrm{~s} . \lambda_{\mathrm{ex}}=532 \mathrm{~nm}, \lambda_{\mathrm{em}}=555-635 \mathrm{~nm}$. Scale bars: $10 \mu \mathrm{m}$.
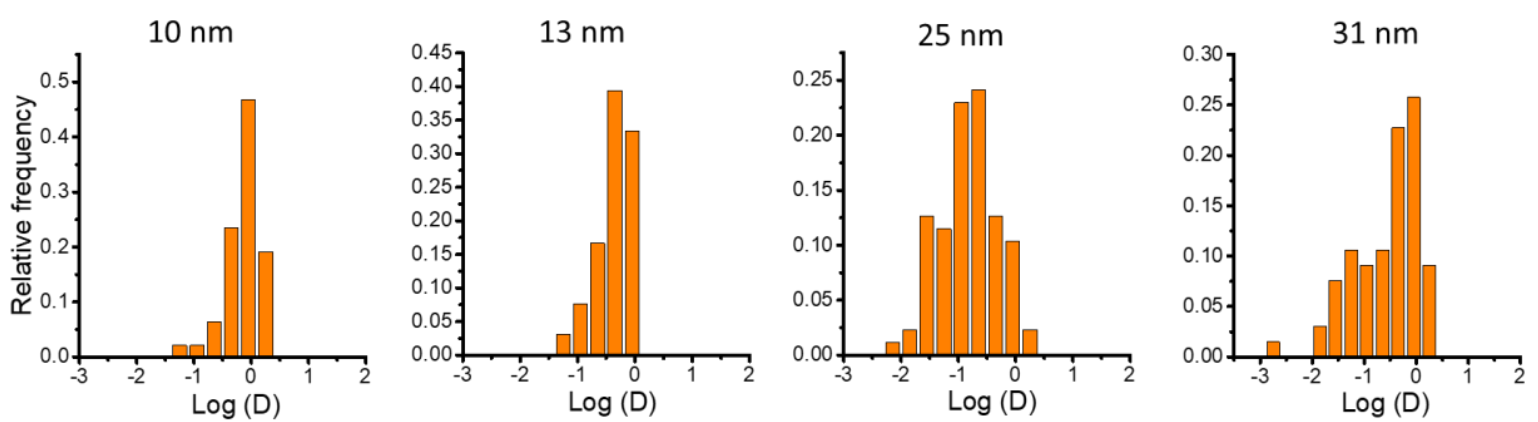

Figure S6. Distribution of diffusion coefficients determined for NPs of different sizes in HeLa cells. At least 30 trajectories were analyzed per sample. 


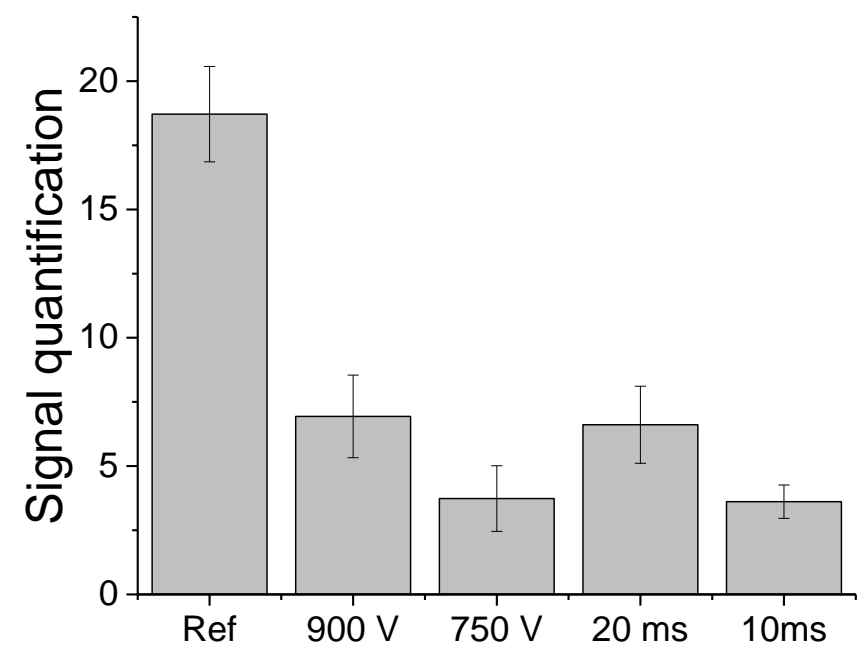

Figure S7. Average fluorescence intensity per cell obtained for different electroporation conditions with $13 \mathrm{~nm}$ NPs. Reference conditions are 2 pulses of $35 \mathrm{~ms}$ with $1050 \mathrm{~V}$. Either pulse amplitude or length were varied as noted. At least 20 cells per condition were evaluated.

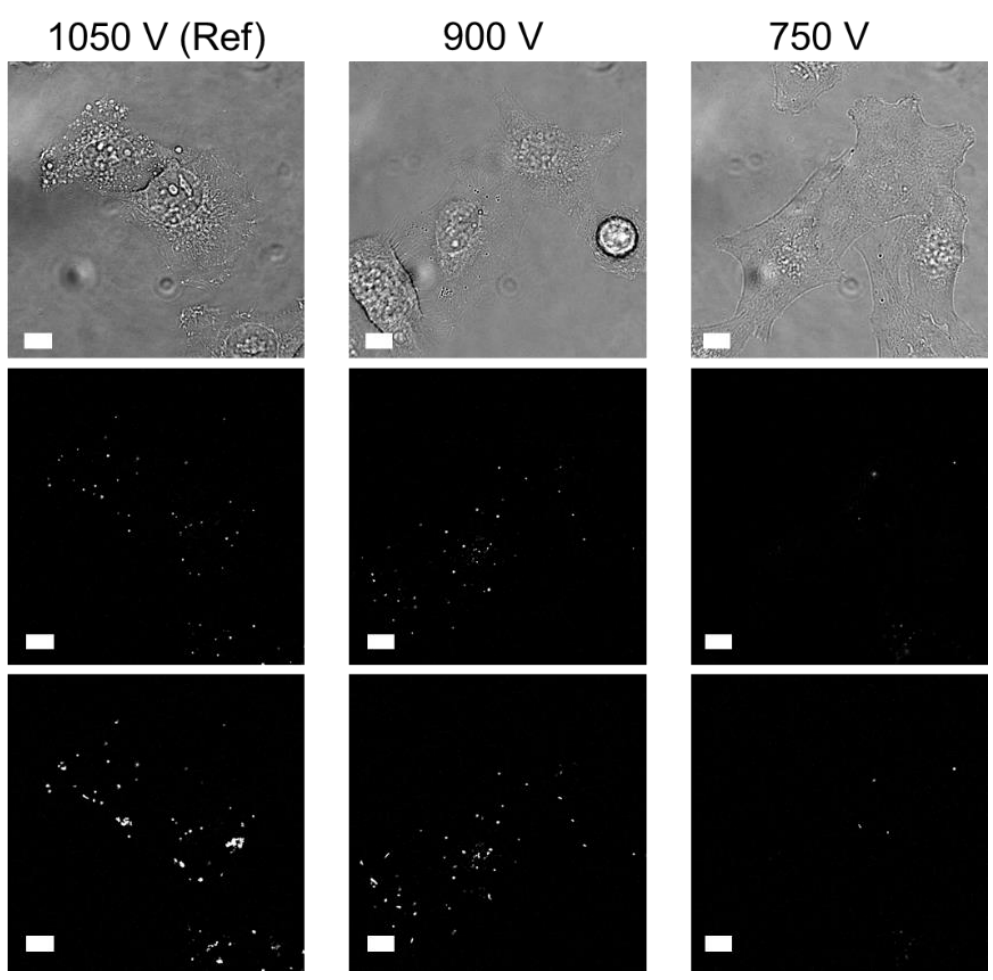

Figure S8. Micrographs of cells electroporated using different pulse intensities with $38 \mathrm{~nm}$ NPs. Top: DIC. Middle: Instantaneous fluorescence image. Bottom: Maximum projections over $36 \mathrm{~s}$. $\lambda_{\text {ex }}=550$ $\mathrm{nm}, \lambda_{\mathrm{em}}=555-635 \mathrm{~nm}$. Scale bars: $10 \mu \mathrm{m}$. 